\title{
Pericarditis Constrictiva, siempre un desafío diagnóstico
}

\author{
Julián Vega, René Hameau, Samuel Córdova, Luigi Gabrielli, Rodrigo Saavedra, Paul McNab, Ricardo, Zalaquett. \\ División de Enfermedades Cardiovasculares. Pontificia Universidad Católica de Chile.
}

Resumen: Reportamos el caso de un hombre de 52 años, con antecedente de larga data de dolor torácico, estudiado el año 2005 con una coronariografía que descarta lesiones en las arterias epicárdicas. Se agrega luego de forma progresiva dolor y distensión abdominal. Sin controles hasta el año 2015, cuando se realiza una endoscopia digestiva alta que no objetiva hallazgos patológicos, encontrándose el paciente en fibrilación auricular e iniciando terapia. A fines del 2016 por persistencia y aumento de las molestias se hospitaliza en nuestro centro para estudio. Se realiza una ecocardiografía que evidencia; severa dilatación bi-auricular, buena función global y segmentaria bi-ventricular y un movimiento de rebote protodiástolico que aumentaba notoriamente en inspiración a nivel del septum interventricular. El pericardio se encontraba engrosado e hiperrefringente de forma difusa, con falta de deslizamiento entre sus hojas y movimiento en bloque de los tejidos blandos adyacentes. Presentaba además comportamiento reverso del anillo mitral al Doppler tisular y la vena cava inferior se encontraba dilatada y sin variación con el ciclo ventilatorio. Un sondeo cardíaco izquierdo y derecho descarta lesiones coronarias y corrobora la fisiología constrictiva. Se amplía el estudio con una tomografía computada de tórax que informa engrosamiento difuso y extensa calcificación del pericardio, además de signos de daño hepático crónico. Se realiza una pericardiotomia exitosa, los hallazgos del intraoperatorio e histopatológicos confirman el diagnóstico de pericarditis constrictiva calcificada. El paciente es dado de alta al quinto día postoperatorio sin incidentes. 


\section{Constrictive Pericarditis. Always a diagnostic challenge}

We report the case of a 52 y.o. male, with longstanding history of chest pain. Coronariography performed in 2005 showed normal coronary arteries. In the following years he developed progressive abdominal bloating and pain, upper gastrointestinal endoscopy was normal, but atrial fibrillation was diagnosed initiating therapy. During the year 2016 symptoms got worse, being admitted to our hospital. Echocardiography revealed severe biatrial enlargement, preserved bi-ventricular function, and a paradoxical bouncing motion of the interventricular septum which augmented during inspiration (septal bounce). The pericardium was thickened with absence of normal sliding between its layers, and trac- tion of adjacent soft tissues was observed. Mitral ring tissue Doppler showed reversal of lateral and medial e' velocities. Inferior vena cava was dilated with no respiratory variation. Cardiac catheterization was consistent with constrictive pericarditis. Computed tomography confirm extensive pericardial calcification and thickness and found signs of chronic liver disease. Uneventful pericardiectomy was performed, intraoperative and histological findings confirm the diagnostic of calcified pericardial constriction, being discharged on the fifth day post-surgery.

Key words: Pericarditis, Constrictive. Heart failure. Pericardiectomy 


\section{Caso}

Reportamos el caso de un hombre de 52 años, con el antecedente de larga data de dolor torácico frente a esfuerzos moderados y que cedía con reposo, estudiado el año 2005 con un test de esfuerzo que resulta positivo para insuficiencia del riego coronario por infradesnivel del segmento ST, realizándose una coronariografía que descarta lesiones en las arterias epicárdicas. Permanece sin controles hasta el año 2015, cuando nota aparición progresiva de distensión y dolor abdominal, evaluado con endoscopia digestiva alta que no reporta hallazgos patológicos, durante el procedimiento se pesquisa en fibrilación auricular, iniciando Amiodarona y Rivaroxaban. Se realiza una ecocardiografía transtorácica (ETT) que informa: dilatación bi-auricular moderada, buena motilidad global y segmentaria biventricular y ausencia de valvulopatías. Durante el año 2016 las molestias descritas se intensifican; con reaparición del dolor torácico frente a esfuerzos leves (caminar 1 cuadra) y aumento de la distensión abdominal. Hospitalizándose en nuestro centro para estudio. Al ingreso se presenta eupneico, normotenso, en fibrilación auricular con FC 90 1.p.m., yugulares ingurgitadas a $45^{\circ}$, con parcial colapso inspiratorio y sin otros hallazgos relevantes.

Los exámenes de laboratorio estaban dentro de rangos normales (hemograma, perfil bioquímico, función renal y perfil hepático). Se inicia el estudio con una ecocardiografía transtorácica (ETT), que debido a la muy mala calidad de las ventanas acústicas, se complementa con una ecocardiografía transesofágica (ETE) (Imagen 1 panel izquierdo), en la que se evidencia una severa dilatación bi-auricular con contraste espontáneo en su interior, un ventrículo izquierdo y derecho de dimensiones normales con buena función global y segmentaria. Distinguiéndose a nivel del septum interventricular un movimiento de "rebote" protodiástolico ("septal bounce"), fenómeno que aumentaba notoriamente en inspiración. El pericardio se encontraba engrosado e hiperrefringente de forma difusa, con falta de deslizamiento entre sus hojas y movimiento en bloque de los tejidos blandos adyacentes al pericardio (Imagen 1 panel derecho). El anillo mitral al Doppler tisular presentaba un comportamiento reverso (mayores velocidades del anillo medial por sobre el lateral) (Imagen 2) y la vena cava inferior se encontraba dilatada y sin variación con el ciclo ventilatorio. La variabilidad de las velocidades en el Doppler transmitral no pudieron ser confiablemente evaluadas por encontrarse el paciente en fibrilación auricular permanente. Con esta fisiología de constricción demostrada por ecocardiografía se realiza

\section{Imagen 1. Ecocardiografía, ETE y ETT.}

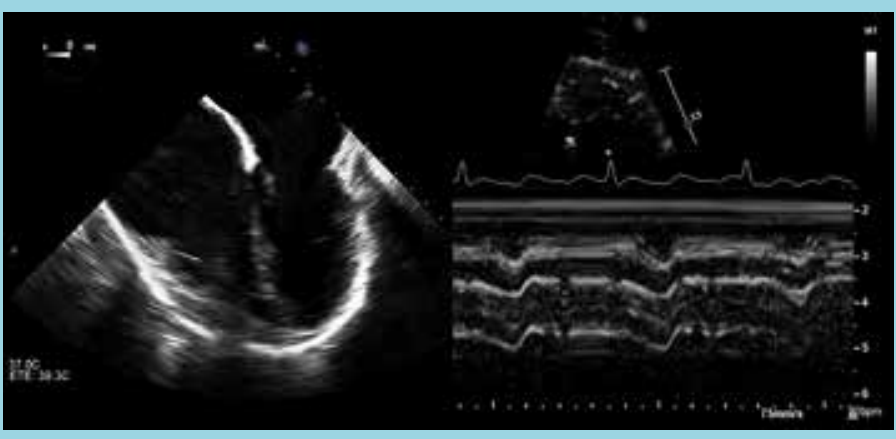

PANEL IZQUIERDO ETE: Visión medio-esofágica de 4 cámaras. Se observa severa dilatación bi-auricular y un pericardio calcificado que rodea todo el corazón.

PANEL DERECHO: ETT, Zoom en segmentos apicales del ventrículo izquierdo desde una visión apical de 4 cámaras. Se observa al modo M movimiento en "bloque" del pericardio junto a la pared ventricular y tejidos adyacentes.

un sondeo cardíaco izquierdo y derecho que descartan lesiones en las arterias epicárdicas y corroboran la fisiología constrictiva; observándose ecualización de las presiones diastólicas en las cuatro cámaras cardíacas, morfología de "W" en la curva de presión auricular derecha, presión del ventrículo derecho con diastólica $>1 / 3$ de la sistólica y curvas ventriculares con configuración de "raíz cuadrada" (“dip-and-plateau”) (Imagen 3). La radiografía de tórax evidencia extensas calcificaciones sobreproyectadas al pericardio sin elementos patológicos en el parénquima pulmonar (Imagen 4, panel izquierdo).

Imagen 2. Doppler Tisular efectuado en QLAB de Philips.

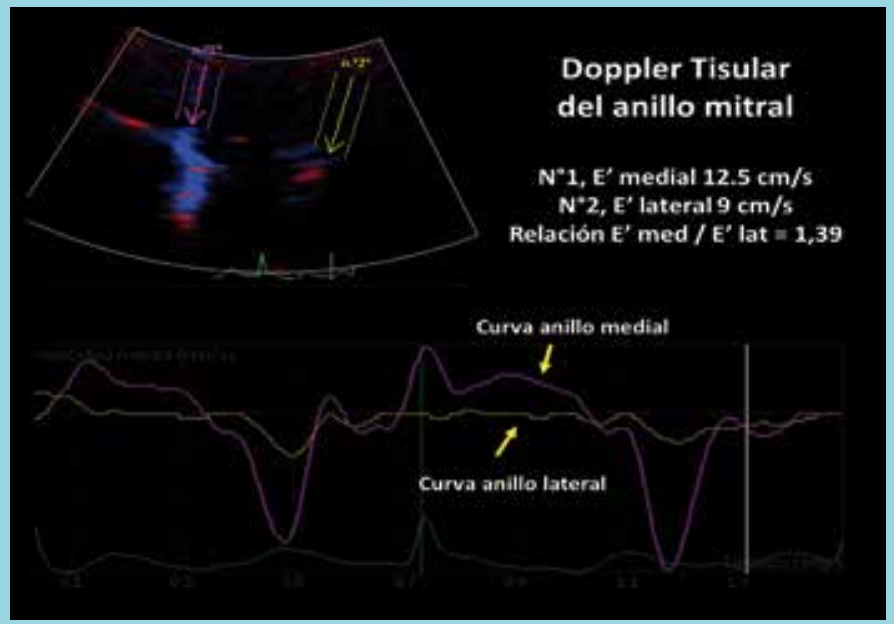

Análisis del movimiento del anillo mitral en el mismo latido. PANEL INFERIOR: Se observa mayor velocidad de la onda E' en el anillo medial (curva morada) por sobre el anillo lateral (curva amarilla), fenómeno denominado anillo reverso. 

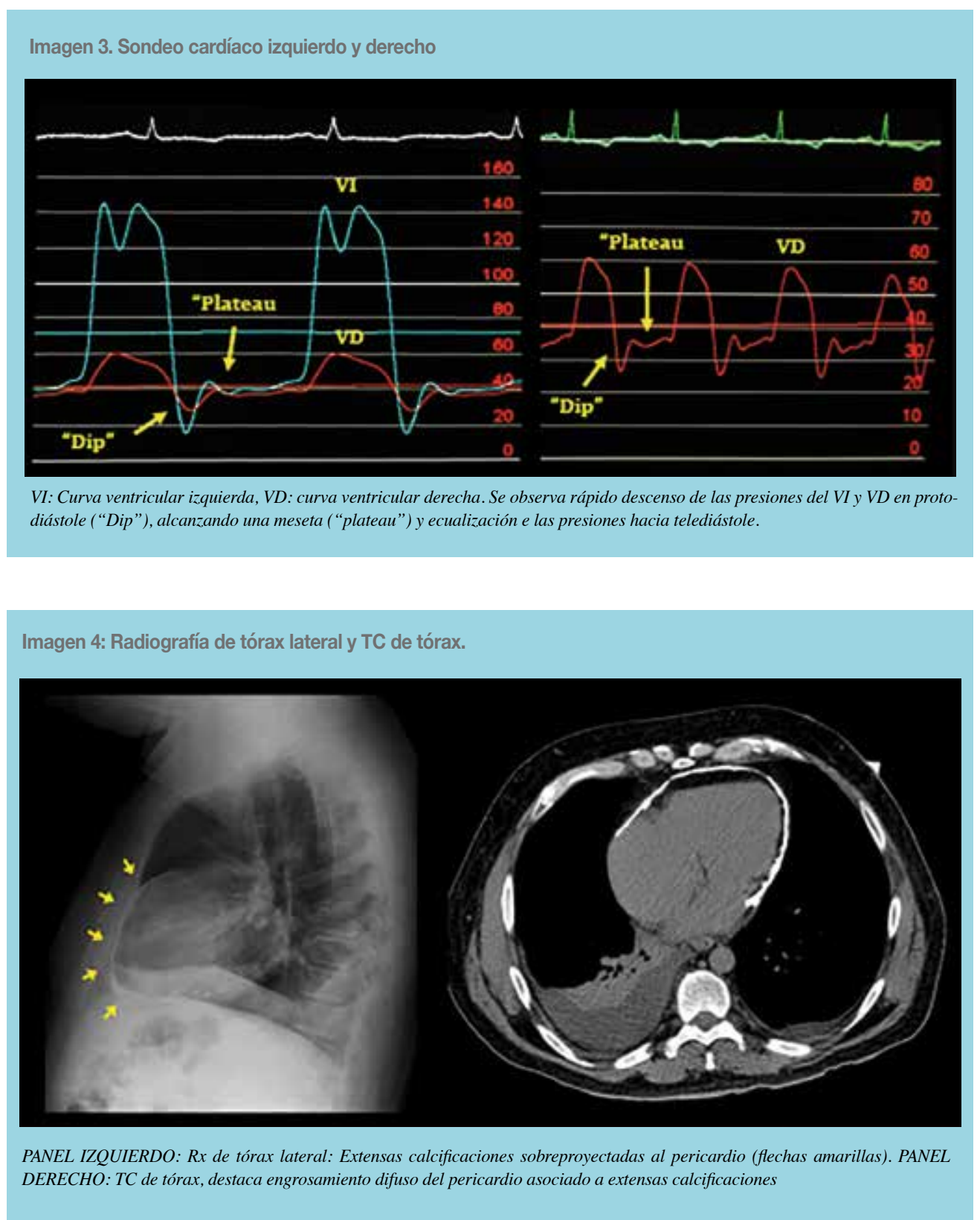

Con el propósito de planificar una pericardiectomía se realiza una tomografía computada (TC) de tórax (Imagen 4, panel derecho), que objetiva un engrosamiento difuso del pericardio con extensas calcificaciones gruesas y en algunas áreas de morfología nodular. Además de signos de daño hepático crónico (prominencia del lóbulo caudado, ensanchamiento cisural y nodularidad de sus contornos). Los hallazgos del intraoperatorio confirmaron un pericardio severamente fibrosado y calcificado (Imagen 5 y video del intraoperatorio y material complementario disponible en http://www.ecocardio.cl/caso_constric- cion.html). Se efectúa una pericardiectomía sin apoyo de circulación extracorpórea, resecando todo el pericardio anterior, inferior y lateral, hasta aproximadamente un centímetro de ambos nervios frénicos, liberando en su totalidad ambas venas cavas, procedimiento sin incidentes. El ETE intraoperatorio al finalizar la cirugía y bajo condiciones hemodinámicas estables, evidencia la desaparición del "septal bounce". La histopatología concluye extensas áreas de calcificación, escasos focos de infiltrado linfocítico y descarta la presencia de granulomas. La recuperación fue satisfactoria, siendo dado de alta al 


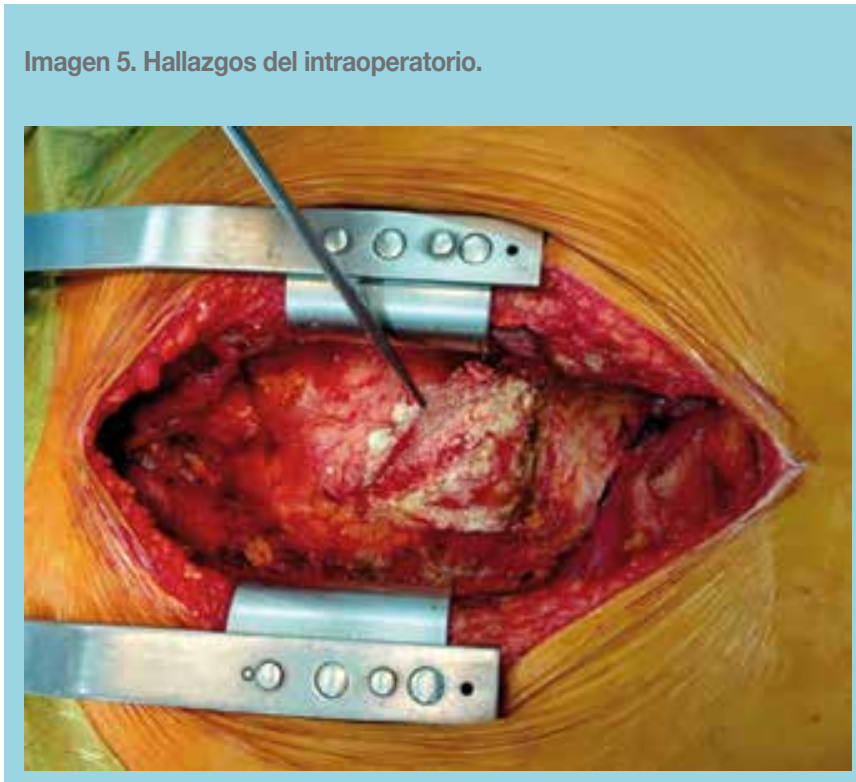

Extensa calcificación y engrosamiento pericárdico con fusión de ambas hojas. Vídeo del intraoperatorio y material complementario disponible en http://www.ecocardio.cl/caso_constriccion.html

quinto día postoperatorio, encontrándose actualmente en capacidad funcional I NYHA.

\section{Discusión}

Dentro de los síndromes de constricción pericárdica se encuentra la pericarditis constrictiva crónica, entidad poco frecuente y de difícil diagnóstico. Que se caracteriza por la dificultad en el llenado ventricular hacia el fin de diástole, debido a la compresión del corazón por un pericardio poco distensible. En países desarrollados sus principales causas son la radioterapia, cirugía cardíaca, enfermedades del tejido conectivo y post infecciosas. Sin embargo, hasta en un 50-75\% de los pacientes no se logra identificar causante ${ }^{1}$, situación que se incrementa cuando existe un largo tiempo de evolución y cuando el pericardio se presenta extensamente calcificado, como el caso reportado ${ }^{1}$.

El diagnóstico habitualmente requiere la participación de tres elementos; clínica (falla derecha sin proporción con falla izquierda), fisiología constrictiva (ecocardiografía Doppler y/o sondeo cardíaco) y evaluación del pericardio por alguna técnica de imagen dedicada (Tomografía computada (TC), resonancia nuclear magnética (RNM)). Es importante señalar que puede presentarse fisiología constrictiva en ausencia de engrosamiento pericárdico (10-20\% de los casos $)^{2,3}$ y en ausencia de calcificación pericárdica (según sea la etiología, más relacionada con tuberculosis, pero no exclusiva de dicha entidad), así como el hallazgo de un pericardio engrosado y/o calcificado puede darse sin fisiología de constricción ${ }^{3,4}$.

El diagnóstico no invasivo de constricción pericárdica es siempre un desafío, a los clásicos hallazgos de la ecocardiografía bidimensional y Doppler se han sumado durante los últimos años elementos de deformación miocárdica

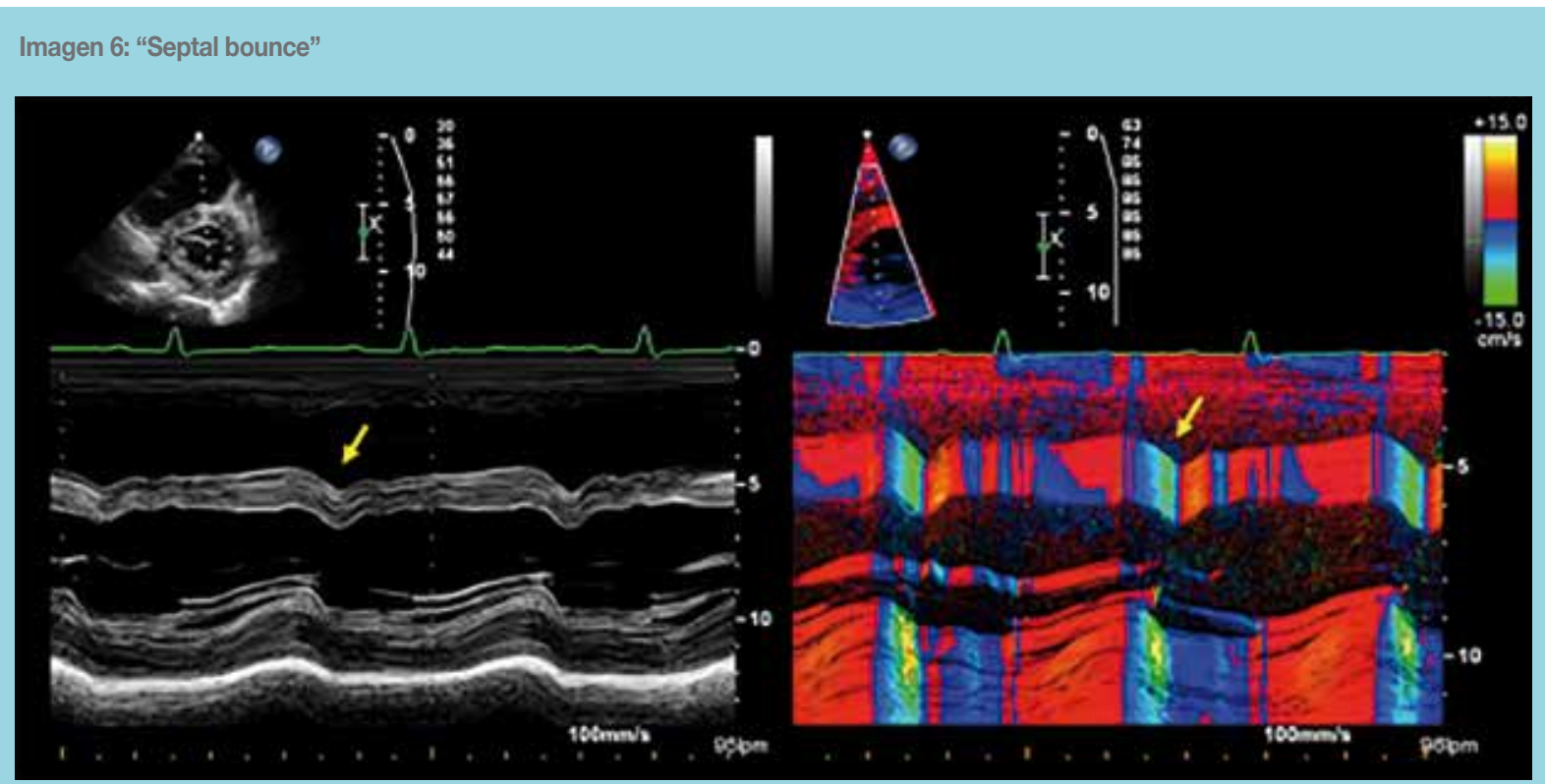

Eje corto paraesternal sobre el ventrículo izquierdo en modo M (panel izquierdo) y modo M tisular (panel derecho). Se observa el "septal bounce" (flecha amarilla), que el modo M tisular lo identifica como un movimiento de alta velocidad (flecha amarilla).

NOTA: Estudio mostrado en esta imagen no corresponde a paciente del caso clínico 


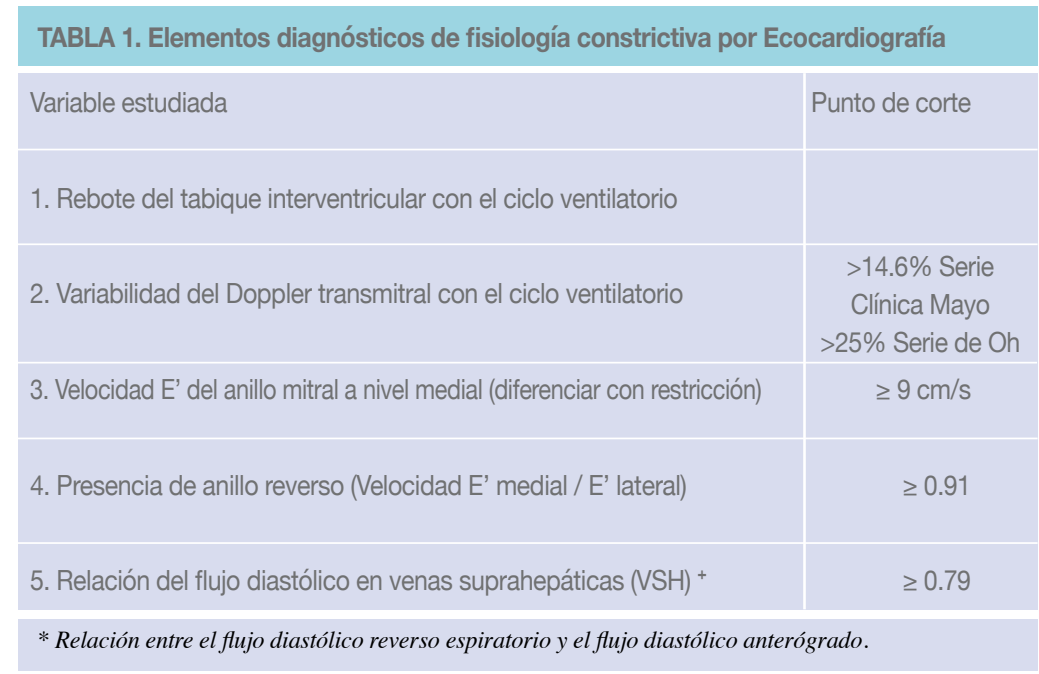

(Doppler tisular y técnicas de deformación ventricular) que mejoran el rendimiento diagnóstico ${ }^{5}$. Las bases del diagnóstico de la fisiología constrictiva, son posibles de demostrar por RNM, TC, ecocardiografía o sondeo cardíaco y, están determinadas por dos fenómenos fisiopatológicos propios de dicho estado ${ }^{6}$;

- Primero, la disociación entre las presiones intratorácicas e intracardíacas, producto de un efecto aislante del pericardio engrosado y rígido.

- Segundo, la presencia de un volumen fijo de fin de diástole, que genera que ambos ventrículos compartan un espacio limitado para llenarse. Este fenómeno es el que origina un marcado aumento en la interdependencia interventricular, que en términos simples, se traduce en que el llenado de un ventrículo impacta el llenado del otro. $\mathrm{Y}$ es esta una de las bases del diagnóstico por ecocardiografía Doppler, que evalúa la disminución del llenado izquierdo (Doppler transmitral) durante la inspiración.

Desde los primeros reportes de la fisiología constrictiva por ecocardiografía Doppler primero por parte de $\mathrm{Hatle}^{7}$ y luego de Jae K. Oh, cerca de los años $90^{8}$, se han publicado diversos elementos diagnósticos ecocardiográficos en constricción. Sin embargo una serie de reciente publicación de la Clínica Mayo ${ }^{9}$ recoge los más relevantes, que se listan en la tabla 1.

En esta serie se destaca como el hallazgo ecocardio- gráfico más consistente y sensible el rebote del tabique interventricular o "septal bounce", que consiste en un movimiento abrupto y de alta velocidad, hacia anterior y luego posterior, que ocurre en protodiástole y que aumenta en inspiración, hallazgo que puede observarse con ecocardiografía bidimensional, modo $\mathrm{M}$ o modo $\mathrm{M}$ tisular (Imagen 6). Y como el hallazgo más específico la relación del flujo diastólico en VSH.

Con relación a la presentación clínica, las clásicas manifestaciones están dadas por aumento de la presión venosa sistémica (edemas periféricos, ascitis, congestión hepática) y disminución del gasto cardíaco (disnea de esfuerzo, intolerancia al ejercicio). Siendo inhabitual la presencia de dolor torácico $(<10 \%)^{10}$ y cambios del ST durante la prueba de esfuerzo, como el caso reportado, fenómeno que puede ser causado por; compresión extrínseca de las arterias coronarias por un pericardio fibrosado ${ }^{11}, 12$, disminución de la perfusión coronaria por bajo gasto cardía$\mathrm{co}^{13}$ y la presencia de enfermedad coronaria asociada ${ }^{14}$. Finalmente la presencia de fibrilación auricular permanente se ha reportado en estados más avanzados de constricción y genera una dificultad diagnóstica añadida, al ser poco fiable las variaciones del Doppler. Siendo claves en dicho escenario; el rebote del tabique, el anillo reverso y la reversión del flujo diastólico en VSH, que por lo demás fueron las únicas variables independientemente asociadas a constricción en la citada serie de la Clínica Mayo $^{9}$. 


\section{Referencias}

1. SAGRISTÀ-SAULEDA J. Síndromes de constricción cardiaca. Rev Española Cardiol. 2008;61:33-40.

2. TALREJA DR, EDWARDS WD, DANIELSON GK, SCHAFF H V., TAJIK AJ, TAZELAAR HD, et al. Constrictive Pericarditis in 26 Patients With Histologically Normal Pericardial Thickness. Circulation. 2003;108:1852-7.

3. NAPOLITANO G, PRESSACCO J, PAQUET E. Cardiovascular radiology: Imaging features of constrictive pericarditis: Beyond pericardial thickening. Can Assoc Radiol J. 2009;60:40-6.

4. NISHIMURA RA. Constrictive pericarditis in the modern era: a diagnostic dilemma. Heart. 2001;86:619-23.

5. DAL-BIANCO JP, SENGUPTA PP, MOOKADAM F, CHANDRASEKARAN K, TAJIK AJ, KHANDHERIA BK. Role of Echocardiography in the Diagnosis of Constrictive Pericarditis. J Am Soc Echocardiogr. 2009;22:24-33.

6. SOHN DW. Constrictive pericarditis as a never ending story: What's new? Korean Circ J. 2012;42:143-50.

7. HATLE LK, APPLETON CP, POPP RL. Differentiation of constrictive pericarditis and restrictive cardiomyopathy by Doppler echocardiography. Circulation. 1989;79:357-70.
8. OH JK, HATLE LK, SEWARD JB, DANIELSON GK, SCHAFF H V, REEDER GS, et al. Diagnostic role of Doppler echocardiography in constrictive pericarditis. J Am Coll Cardiol. 1994;23:154-62.

9. WELCH TD, LING LH, ESPINOSA RE, ANAVEKAR NS, WISTE HJ, LAHR BD, et al. Echocardiographic diagnosis of constrictive pericarditis Mayo Clinic criteria. Circ Cardiovasc Imaging. 2014;7:526-34.

10. LING LH, OH JK, SCHAFF H V, DANIELSON GK, DOUGLAS W, SEWARD JB, et al. Constrictive Pericarditis in the Modern Era. Circulation. 1999;100:1380-6.

11. MUKHOPADHYAY S, YUSUF J, GIRISH MP, GUPTA MD, TREHAN V. Coronary constriction in constrictive pericarditis. Int J Cardiol. 2006;106:135-6.

12. SOMA K, TAKAHASHI M, SATO T, NAGAI R. Angina as a Manifestation of Constrictive Pericarditis. Intern Med. 2012;51:1939-1939.

13. TOPAZ O, NAIR R, MACKALL JA. Observations of angina and myocardial infarction in constrictive pericarditis. Int $\mathrm{J}$ Cardiol. 1993;39:121-9.

14. TRIBAK M, KONATÉ M, BENOMAR M. Association of severe class III angina pectoris and constrictive pericarditis. Arch Cardiovasc Dis. 2008;101:196-7. 